\title{
Relationship between Childhood Maltreatment, Suicidality, and Bipolarity: A Retrospective Study
}

\author{
Young-Min Park $\bowtie$ \\ Department of Psychiatry, Ilsan Paik Hospital, Inje University College of Medicine, Goyang, Republic of Korea
}

\begin{abstract}
Objective The aims of current study were to determine whether childhood maltreatment contributes to the occurrence of major depressive disorder (MDD) with bipolarity or suicidality.

Methods In total, 132 outpatients diagnosed with MDD between 2014 and 2015 on the medical records were included. The subjects were divided into two groups according to the presence of childhood maltreatment (CM group) and no childhood maltreatment (NCM group). Depression severity and bipolarity were identified using Beck Depression Inventory (BDI) and the Korean version of Mood Disorder Questionnaire (K-MDQ) respectively on the medical records. In addition, the baseline loud dependence of auditory evoked potentials of 36 patients on medical records were analyzed.

Results The mean total BDI, BDI item 9 (suicide ideation), and total K-MDQ score were significantly higher in the CM group than the NCM group. The number of subjects with bipolarity was significantly higher in the CM than in the NCM group. Furthermore two thirds subjects experienced the significant maltreatment during childhood. The central serotonergic activity of the CM group was also lower than that of the NCM group.

Conclusion The findings of this study support that there is a relationship between childhood maltreatment and bipolarity or suicidality in patients with MDD.

Psychiatry Investig 2017;14(2):136-140
\end{abstract}

Key Words Bipolarity, Childhood maltreatment, Trauma, LDAEP, Major depressive disorder, Bipolar disorder.

\section{INTRODUCTION}

Childhood abuse is the one of notorious social issues, causing the most severe physical and mental health problems. ${ }^{1}$ It significantly influences the mental health not only in childhood but also in later adulthood. ${ }^{2}$ Thus, it can cause people to suffer from mental disorder such as mood disorder, anxiety disorder, and alcohol in adulthood. ${ }^{3,4}$ Especially, strong evidence from some prospective or cross-sectional study reflects that childhood abuse is significantly related to the onset of MDD in both adolescence and adulthood. ${ }^{5,6}$ A recent metaanalysis also revealed that individuals with the early experience of abuse or neglect have a higher risk of developing depressive disorders than nonabused individuals. ${ }^{2}$ Moreover, the

Received: March 15, 2016 Revised: June 25, 2016

Accepted: July 10, 2016 Available online: January 6, 2017

$\triangle$ Correspondence: Young-Min Park, MD, PhD

Department of Psychiatry, Ilsan Paik Hospital, Inje University College of Medicine, 170 Juhwa-ro, Ilsansu-gu, Goyang 10380, Republic of Korea

Tel: +82-31-910-7260, Fax: +82-31-910-7268, E-mail: medipark@hanmail.net

(a) This is an Open Access article distributed under the terms of the Creative Commons Attribution Non-Commercial License (http://creativecommons.org/licenses/bync/4.0) which permits unrestricted non-commercial use, distribution, and reproduction in any medium, provided the original work is properly cited. short variant of the serotonin transporter gene made people vulnerable to the effect of childhood abuse or neglect and caused their adult depressive episodes to a chronic course in some studies. ${ }^{7}$

Li et al. ${ }^{8}$ found that patients with BD reported also reported their childhood abuse and neglect. In addition, childhood abuse is associated with bipolar disorder and its clinical expression and may interact with genetic susceptibility factors. ${ }^{9}$ However, there are still few studies about the relationship between childhood abuse and bipolar disorder. Furthermore, to date there have been no reports of a relationship between childhood abuse and MDD with bipolarity or bipolar spectrum disorder, which has no manic or hypomanic episode but has the characteristics of $\mathrm{BD} .^{10}$

It was hypothesized that childhood adversity like abuse or neglect produce more bipolarity in MDD. More specifically, the aims of the current study were to determine whether childhood adversity contributes to the occurrence of MDD with bipolarity, and whether there is a relationship between childhood adversity and central serotonergic activity, as reflected by loudness dependence of auditory evoked potentials (LDAEP), which is measured using auditory processing, has recently 
been introduced as a metric of serotonin activity. ${ }^{11}$ The LDAEP has been identified as being inversely associated with central serotonergic activity, with a large LDAEP reflecting low serotonergic neurotransmission and vice versa. ${ }^{12}$ The current study is a respective study, using the existence of childhood maltreatment and bipolarity based on medical records.

\section{METHODS}

In total, 132 outpatients aged between 20 and 65 years who met the Diagnostic and Statistical Manual of Mental Disorders (DSM-IV)-text revision criteria for MDD were included at Ilsan Paik Hospital between 2014 and 2015 on the medical records. Subjects who had psychotic symptoms, any additional mental disorders on axis I or II of the DSM-IV were excluded to remove bias. None of the subjects had a history of hypomanic or manic episodes. In addition, the subjects had no history of antidepressant treatment within 1 week before first visiting our hospital. The baseline LDAEP of 62 subjects, which was used as a marker of serotonergic activity in our previous study, ${ }^{13}$ were collected in case of existing on medical records for further analysis, but finally, the baseline LDAEP of 36 patients, who revealed suicide ideation and $\geq 20$ total BDI score, were selected for the analysis of homogenous data.

The subjects were divided into two groups according to the presence of childhood maltreatment (CM group) and no childhood maltreat (NCM group), using the Korean version of the Childhood Trauma Questionnaire (K-CTQ) ${ }^{14,15}$ which indicated subjects as positive for a history of childhood abuse or neglect based on the cutoff score of the total K-CTQ (i.e.,
41 points). ${ }^{16}$ The K-CTQ is a self-report questionnaire that have five subscales of childhood abuse or neglect experience: physical abuse (PA), emotional abuse (EA), sexual abuse (SA), physical neglect (PN), and emotional neglect (EN). Each subscale consists of five items that are rated on a 5-point scale, from 1 (never true) to 5 (very often true). The subjects were also divided into the following two groups according to whether they had suffered no maltreatment or had suffered one or more types of maltreatment (i.e., no vs one or more maltreatments) based on the moderate-to-severe cutoff scores of five subscales: EA, $\geq 13$; PA, $\geq 10$; $\mathrm{SA}, \geq 8$; EN, $\geq 15$; and $\mathrm{PN}$, $\geq 10 .{ }^{16}$ Depression severity and bipolarity were assessed using Beck Depression Inventory (BDI) and the Korean version of Mood Disorder Questionnaire (K-MDQ) respectively on the medical records. The K-MDQ consists of three main parts. In the first part, the K-MDQ questions about the existence of manic or hypomanic symptoms using 13 items. In the second part, it does whether these manic or hypomanic symptoms have been experienced at the same time, and in the last part, it also does the functional impairment due to these symptoms. ${ }^{17} \mathrm{~K}-\mathrm{MDQ}$ indicated subjects as positive for a screening of bipolarity based on a cutoff score of total K-MDQ (i.e., 7 points). ${ }^{17}$ Based on this cutoff score, the subjects were divided into two groups indicating the presence of subthreshold bipolarity.

The study protocol was approved by the ethics committee of Inje University Ilsan Paik Hospital, and written informed consent to participate was obtained from all patients before beginning the investigation.

Table 1. Comparison of demographic and clinical variables between the childhood maltreatment (CM) and no childhood maltreatment (NCT) groups, defined according to the cutoff (41 points) for the total Korean version of the Childhood Trauma Scale (K-CTQ) Score

\begin{tabular}{lccc}
\hline \multicolumn{1}{c}{ Variable } & NCM $(\mathrm{N}=44)$ & $\mathrm{CM}(\mathrm{N}=88)$ & $\mathrm{p}$ \\
\hline Age (years) & $34.84 \pm 11.03$ & $32.61 \pm 10.35$ & 0.26 \\
Sex (male/female) ${ }^{\dagger}$ & $15 / 29$ & $33 / 55$ & 0.70 \\
Total BDI score & $20.09 \pm 11.99$ & $25.26 \pm 10.11$ & $<0.05^{*}$ \\
BDI item 9 score & $0.48 \pm 0.79$ & $0.79 \pm 0.80$ & $<0.05^{*}$ \\
Total K-MDQ score & $3.93 \pm 2.99$ & $6.52 \pm 3.44$ & $<0.01^{* *}$ \\
Bipolarity (no/yes) ${ }^{\dagger}$ & $34 / 10$ & $44 / 44$ & $<0.01^{* *}$ \\
Total CTQ score & $31.58 \pm 4.59$ & $57.18 \pm 15.50$ & $<0.01^{* *}$ \\
EN score & $7.57 \pm 3.26$ & $16.35 \pm 4.88$ & $<0.01^{* *}$ \\
PA score & $6.09 \pm 1.87$ & $11.08 \pm 5.6$ & $<0.01^{* *}$ \\
SA score & $5.25 \pm 0.69$ & $7.33 \pm 4.08$ & $<0.01^{* *}$ \\
EA score & $5.61 \pm 1.24$ & $11.53 \pm 4.84$ & $<0.01^{* *}$ \\
PN score & $7.06 \pm 2.21$ & $10.89 \pm 4.01$ & $<0.01^{* *}$ \\
\hline
\end{tabular}

Comparisons were made by independent $t$ test. ${ }^{*} \mathrm{p}<0.05,{ }^{* *} \mathrm{p}<0.01,{ }^{\dagger}$ chi-square test. BDI: Beck Depressive Inventory, K-MDQ: Korean version of Mood Disorder Questionnaire, EN: emotional neglect, EA: emotional abuse, PA: physical abuse, PN: physical neglect, SA: sexual abuse 


\section{RESULTS}

A comparison of the demographic and clinical variables between the CM and NCM groups, defined according to the total K-CTQ score, is presented in Table 1. The mean total BDI, BDI item 9 (suicide idea), and total K-MDQ score differed significantly between the NCM group and the CM group. The mean scores of all the K-CTQ subscales were significantly higher in the CM than in the NCM group. The number of subjects with bipolarity was significantly higher in the CM than in the NCM group. In addition, two thirds subjects experienced the significant maltreatment in childhood.

Table 2 presents the demographic and clinical variables among the subjects stratified according to bipolarity, defined according to the cutoff score for K-MDQ. The mean total BDI, K-CTQ, PA, SA, EA scores were significantly higher in the group with bipolarity than in that without bipolarity. The number of subjects with the experience of childhood maltreat-

Table 2. Comparison of demographic and clinical variables between the bipolarity and nonbipolarity groups, defined according to the cutoff (7 points) for the total K-MDQ score

\begin{tabular}{lccc}
\hline \multicolumn{1}{c}{ Variable } & Bipolarity $(-)(\mathrm{N}=78)$ & Bipolarity $(+)(\mathrm{N}=54)$ & $\mathrm{p}$ \\
\hline Age (years) & $34.08 \pm 10.40$ & $32.31 \pm 10.88$ & 0.35 \\
Sex (male/female) ${ }^{\dagger}$ & $24 / 54$ & $24 / 30$ & 0.11 \\
Total BDI score & $21.23 \pm 11.28$ & $26.87 \pm 9.75$ & $<0.01^{* *}$ \\
BDI item 9 score & $0.63 \pm 0.76$ & $0.77 \pm 0.87$ & 0.33 \\
Total K-MDQ score & $3.23 \pm 1.97$ & $9.17 \pm 1.87$ & $<0.01^{* *}$ \\
K-CTQ (negative/positive) ${ }^{\dagger}$ & $34 / 44$ & $10 / 44$ & $<0.01^{* *}$ \\
Total CTQ score & $45.13 \pm 16.88$ & $53.71 \pm 17.77$ & $<0.01^{* *}$ \\
EN score & $12.74 \pm 6.25$ & $14.41 \pm 5.67$ & 0.12 \\
PA score & $8.58 \pm 4.69$ & $10.63 \pm 5.79$ & $<0.05^{*}$ \\
SA score & $6.13 \pm 2.77$ & $7.37 \pm 4.24$ & $<0.05^{*}$ \\
EA score & $8.37 \pm 4.62$ & $11.28 \pm 4.79$ & $<0.01^{* *}$ \\
PN score & $9.31 \pm 3.94$ & $10.03 \pm 3.95$ & 0.30 \\
\hline
\end{tabular}

Comparisons were made by independent $\mathrm{t}$ test. ${ }^{*} \mathrm{p}<0.05,{ }^{* *} \mathrm{p}<0.01,{ }^{\dagger}$ chi-square test. BDI: Beck Depressive Inventory, K-MDQ: Korean version of Mood Disorder Questionnaire, K-CTQ: Korean version of the Childhood Trauma Scale, EN: emotional neglect, EA: emotional abuse, PA: physical abuse, PN: physical neglect, SA: sexual abuse

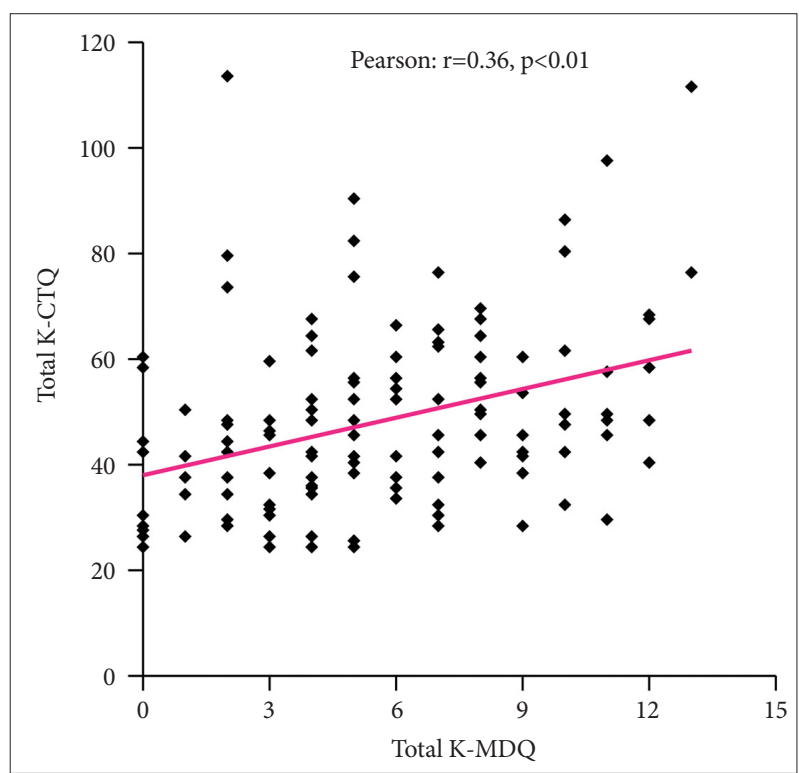

Figure 1. Correlation between the Korean version of the Childhood Trauma Questionnaire (K-CTQ) and the Korean version of Mood Disorder Questionnaire (K-MDQ). The total K-CTQ score was positively correlated with the total $K-M D Q$ score $(r=0.36$, $\mathrm{p}<0.01)$.

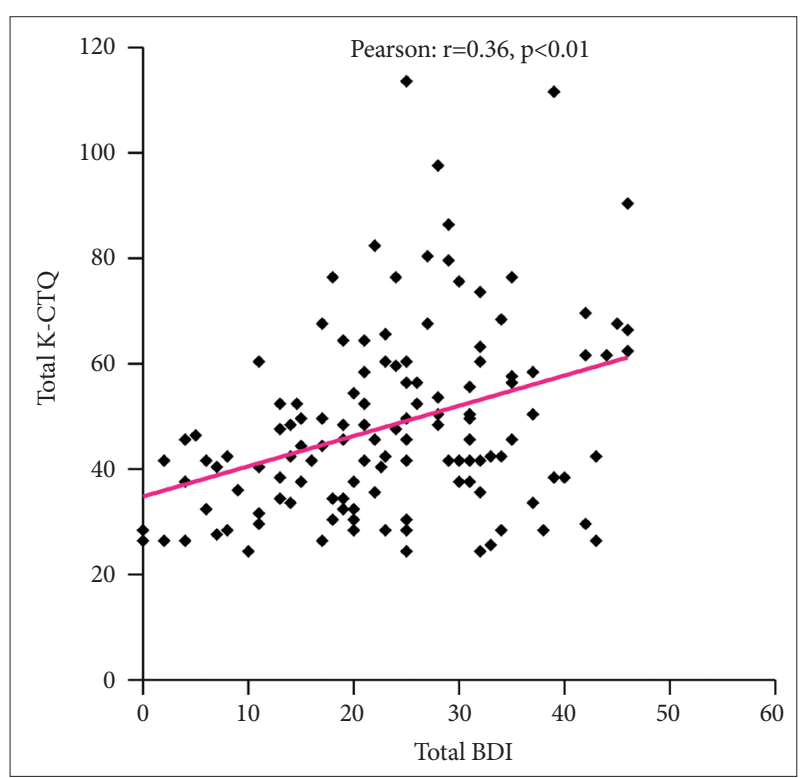

Figure 2. Correlation between the Korean version of the Childhood Trauma Questionnaire (K-CTQ) and the Beck Depression Inventory (BDI). The total K-CTQ score was positively correlated with the total BDI score $(r=0.36, p<0.01)$. 
ment was significantly higher in the bipolarity than in the nonbipolarity group.

The total K-CTQ score was positively correlated with the total K-MDQ and Total BDI score (Figures 1 and 2). All the subscales of K-CTQ were positively correlated with the BDI item 9 and with the total BDI except SA and with K-MDQ scores except PN. Logistic regression indicated the significant association between EN, EA, SA, PA, and bipolarity (K-MDQ score).

Further analysis of the group $(\mathrm{n}=36)$ having the LDAEP value in the medical records was performed. The mean LDAEP value differed significantly between the NCM group and the CM group $(t=-2.09, d f=34, p=0.044)$ (Figure 3 ). In other words, the LDAEP of the CM group was higher than that of the NCM group. In addition, there was a positive correlation between LDAEP and PN score $(r=0.39, \mathrm{p}=0.019)$. The K-MDQ score was also positively correlated with SA and BDI item 9 (suicide ideation) score.

\section{DISCUSSION}

Our retrospective data indicated the relationship between childhood maltreatment and bipolarity or suicidality in patients with MDD. In other words, the subjects of the CM group had higher scores of K-MDQ and the suicide item on BDI than did those in the NCM group. Especially, most of the subscales of K-CTQ such as PA, SA, EA, and EN were positively correlated with the BDI item 9 and K-MDQ. These mean that childhood maltreatment is associated with both

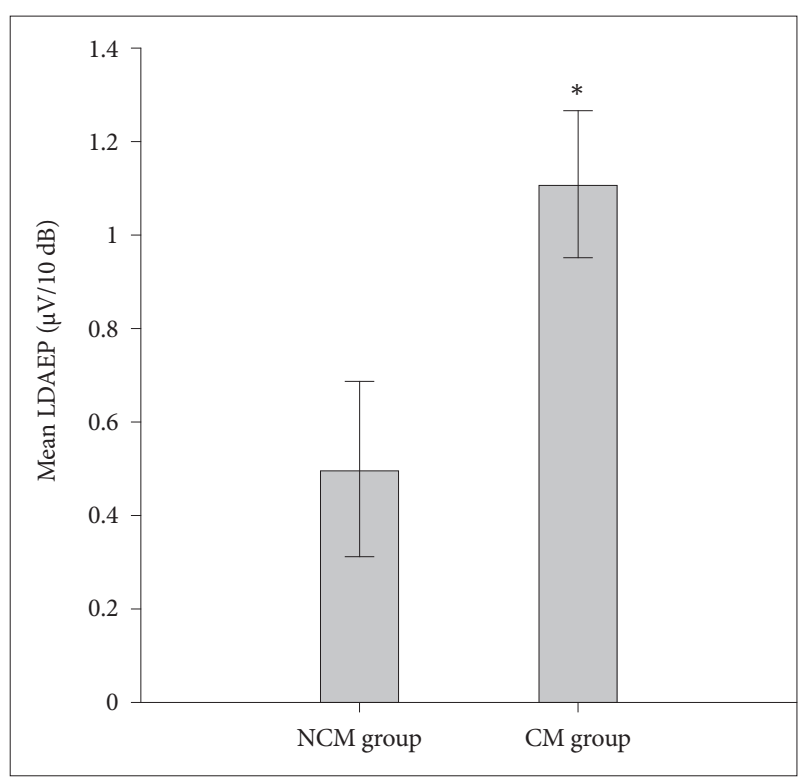

Figure 3. Comparison in LDAEP between no childhood maltreatment group (NCM) and childhood maltreatment group (CM). *the mean LDAEP value differed significantly between the NCM group and the CM group $(t=-2.09, d f=34, p=0.044)$. increased risk of bipolarity and suicidality. Our previous pilot cross-sectional study also indicated a relationship between childhood maltreatment and bipolarity or suicidality in patients with MDD. ${ }^{6}$ However, this pilot study had a very small sample size $(n=35)$ as limitation. Thus, the current study seems to replicate our previous results and strengthen this hypothesis that childhood maltreatment potentiates the bipolarity and suicidality in MDD patients. Previous studies have demonstrated that childhood maltreatment was associated with the increased risk for mood disorder. ${ }^{3,9,18}$ Some investigators reported that childhood maltreatment increased risk for lifetime depressive disorders. ${ }^{18}$ Others reported that a significant number of subjects with BD indicated childhood maltreatment. ${ }^{8}$ Recently, the exposure to childhood maltreatment potentiated the effects of recent stressors on adulthood mania. ${ }^{19}$ Thus, childhood maltreatment seems to increase the risk for mood disorder such as bipolar disorder. However, our results are so unique in that they are specific to MDD patients with increased bipolarity not BD. Thus, it is also possible that childhood maltreatment increase the severity of MDD such as impulsiveness, affective instability, and suicidality as well as the bipolarity. ${ }^{9}$ Especially, MDQ scale can reflect impulsiveness and affective instability, and borderline personality patients revealed high MDQ scores as well as bipolar patients. ${ }^{20}$ However, because borderline patients were not included in the current study, there was still inconclusive.

Further analysis of the LDAEP group revealed LDAEP of the CM group was higher than that of the NCM group. In other words, central serotonergic activity of the CM group was lower than that of the NCM group. A recent study revealed the relationship of the polymorphisms of the serotonin transporter gene and the effect of childhood maltreatment on adulthood depression. ${ }^{21}$ In addition, the relationship between serotonin gene and SA or PA, has been found in recurrent depressive disorder. ${ }^{7}$ Low serotonergic activity using LDAEP was also associated with suicidality. ${ }^{22}$ In addition, our previous study showed the relationship between LDAEP and bipolarity score. ${ }^{6}$ It has been hypothesized that inappropriate rewards and parental attitudes during childhood induce affective dysregulation in children with brain structural or functional abnormalities such as sertonergic dysfunction. ${ }^{5}$

The small sample size and retrospective study design in the current study are limitations. Especially, this study could rule out PTSD and borderline personality disorder because of a retrospective design. In addition, the K-MDQ is a screening scale to help identify bipolarity, and not a case-finding instrument for bipolarity itself. Some investigators claim that because of the MDQ's modest positive predictive value, most patients who screen positive will not have bipolar disorder. ${ }^{20}$ However, MDQ is also a useful scale except screening the bi- 
polar disorder. Recently, Park and Lee ${ }^{23}$ found that depressed patients with bipolarity had a worse response to antidepressant monotherapy than did those without bipolarity using KMDQ. Moreover, a clinician's prior probability estimate about the likelihood of bipolar disorder in a particular patient-based on a patient's history, family history, past treatment response, or other clinical impressions - has as much or more impact on the performance of the MDQ or BSDS as the test's sensitivity and specificity. ${ }^{24}$

In spite of these limitations, this is the study to evaluate the relationship between childhood maltreatment and bipolarity or suicidality. In conclusion, the findings of this study support that there is a relationship between childhood maltreatment and bipolarity or suicidality in patients with MDD. In future, more studies with larger cohorts are needed to confirm the findings.

\section{Acknowledgments}

This study was supported by a grant from National Research Foundation of Korea (NRF), funded by Ministry of Education (NRF-2014R1A1A2A 10059345). The authors would like to thank Jo BW for her assistance with data collection.

\section{REFERENCES}

1. Pereda N, Guilera G, Forns M, Gomez-Benito J. The prevalence of child sexual abuse in community and student samples: a meta-analysis. Clin Psychol Rev 2009;29:328-338.

2. Norman RE, Byambaa M, De R, Butchart A, Scott J, Vos T. The longterm health consequences of child physical abuse, emotional abuse, and neglect: a systematic review and meta-analysis. PLoS Med 2012; 9:e1001349.

3. Arnow BA. Relationships between childhood maltreatment, adult health and psychiatric outcomes, and medical utilization. J Clin Psychiatry 2004;65(Suppl 12):10-15.

4. Sachs-Ericsson N, Cromer K, Hernandez A, Kendall-Tackett K. A review of childhood abuse, health, and pain-related problems: the role of psychiatric disorders and current life stress. J Trauma Dissociation 2009;10:170-188.

5. Etain B, Henry C, Bellivier F, Mathieu F, Leboyer M. Beyond genetics: childhood affective trauma in bipolar disorder. Bipolar Disord 2008; 10:867-876.

6. Lee BH, Park YM. How childhood maltreatment is related to suicidality, bipolarity and central serotonergic activity in patients with major depressive disorder: a cross-sectional pilot study. Psychiatry Investig 2016;13:190-195.

7. Fisher HL, Cohen-Woods S, Hosang GM, Korszun A, Owen M, Craddock $\mathrm{N}$, et al. Interaction between specific forms of childhood maltreatment and the serotonin transporter gene (5-HTT) in recurrent depressive disorder. J Affect Disord 2013;145:136-141.

8. Li XB, Liu JT, Zhu XZ, Zhang L, Tang YL, Wang CY. Childhood trauma associates with clinical features of bipolar disorder in a sample of Chinese patients. J Affect Disord 2014;168:58-63.

9. Etain B, Mathieu F, Henry C, Raust A, Roy I, Germain A, et al. Preferential association between childhood emotional abuse and bipolar disorder. J Trauma Stress 2010;23:376-383.

10. Ghaemi SN. Bipolar spectrum: a review of the concept and a vision for the future. Psychiatry Investig 2013;10:218-224.

11. Hegerl U, Juckel G. Intensity dependence of auditory evoked potentials as an indicator of central serotonergic neurotransmission: a new hypothesis. Biol Psychiatry 1993;33:173-187.

12. Juckel G, Hegerl U, Molnar M, Csepe V, Karmos G. Auditory evoked potentials reflect serotonergic neuronal activity--a study in behaving cats administered drugs acting on 5-HT1A autoreceptors in the dorsal raphe nucleus. Neuropsychopharmacology 1999;21:710-716.

13. Park YM, Lee SH. Can the loudness dependence of auditory evoked potentials and suicidality be used to differentiate between depressive patients with and without bipolarity. Psychiatry Investig 2013;10:143-147.

14. Bernstein DP, Stein JA, Newcomb MD, Walker E, Pogge D, Ahluvalia T, et al. Development and validation of a brief screening version of the Childhood Trauma Questionnaire. Child Abuse Negl 2003;27:169-190.

15. Yu JH, Park JS, Park DH, Ryu SH, Ha JH. Validation of the Korean childhood trauma questionnaire: the practical use in couselling and therapeutic intervention. Kor J Health Psychol 2009;14:563-578.

16. Bernstein D, Fink L. Childhood Trauma Questionnaire: A Retrospective Self-Report Questionnaire and Manual. San Antonio, TX: Psychological Corp; 1998.

17. Jon DI, Hong N, Yoon BH, Jung HY, Ha K, Shin YC, et al. Validity and reliability of the Korean version of the mood disorder questionnaire. Compr Psychiatry 2009;50:286-291.

18. Chapman DP, Whitfield CL, Felitti VJ, Dube SR, Edwards VJ, Anda RF. Adverse childhood experiences and the risk of depressive disorders in adulthood. J Affect Disord 2004;82:217-225.

19. Gilman SE, Ni MY, Dunn EC, Breslau J, McLaughlin KA, Smoller JW, et al. Contributions of the social environment to first-onset and recurrent mania. Mol Psychiatry 2015;20:329-336.

20. Zimmerman M. Misuse of the mood disorders questionnaire as a casefinding measure and a critique of the concept of using a screening scale for bipolar disorder in psychiatric practice. Bipolar Disord 2012;14:127134.

21. Miller JM, Kinnally EL, Ogden RT, Oquendo MA, Mann JJ, Parsey RV. Reported childhood abuse is associated with low serotonin transporter binding in vivo in major depressive disorder. Synapse 2009;63:565-573.

22. Kim DH, Park YM. The association between suicidality and serotonergic dysfunction in depressed patients. J Affect Disord 2013;148:72-76.

23. Park YM, Lee BH. Treatment response in relation to subthreshold bipolarity in patients with major depressive disorder receiving antidepressant monotherapy: a post hoc data analysis (KOMDD study). Neuropsychiatr Dis Treat 2016;12:1221-1227.

24. Phelps JR, Ghaemi SN. Improving the diagnosis of bipolar disorder: predictive value of screening tests. J Affect Disord 2006;92:141-148. 